\title{
Perfil socioeconômico e satisfação dos pacientes atendidos no mutirão de catarata do Instituto da Visão - UNIFESP
}

\author{
Socioeconomic profile and satisfaction of individuals attended during the cataract \\ project of the Vision Institute-Ophthalmology department-UNIFESP
}

Luci Meire Pereira da Silva ${ }^{1}$

Cristina Muccioli²

Rubens Belfort $\mathbf{J r}^{3}$

\section{RESUMO}

Objetivo: Identificar as características socioeconômicas, dados epidemiológicos e demográficos, bem como avaliar a satisfação dos pacientes com relação ao atendimento recebido durante o Mutirão de Catarata, do Instituto da Visão/Departamento de Oftalmologia - UNIFESP. Método: Durante o Mutirão de Catarata realizado em 05/10/02, pelo Instituto da Visão - UNIFESP, foram selecionados, na fila de espera, alternadamente e aleatoriamente, 133 pacientes, que foram entrevistados e responderam a perguntas de questionários desenvolvidos para identificar o perfil socioeconômico e avaliar a satisfação destes pacientes. Resultados: A amostra, formada por 133 sujeitos, representou $50 \%$ do atendimento e revelou as seguintes características socioeconômicas: 61 (46\%) eram do sexo masculino e 72 (54\%), do sexo feminino; 117 (87\%) tinham mais de 50 anos de idade; $111(84 \%)$ pacientes possuíam nível de instrução apenas até o primeiro grau; $74(55 \%)$ eram aposentados ou pensionistas; 99 (74\%) usufruíam renda familiar de até $\mathrm{R}$ \$ 500,00; 91 (68\%) tiveram necessidade de vir com acompanhante; $95(71 \%)$ dos pacientes apresentavam catarata com indicação cirúrgica. Com relação à satisfação quanto ao atendimento, 122 $(91 \%)$ dos entrevistados, classificaram como boa a qualidade geral do atendimento; $10(8 \%)$, regular e 1(1\%), ruim. Conclusão: A maioria da população atendida no Mutirão de Catarata do Instituto da Visão Departamento de Oftalmologia - UNIFESP ficou satisfeita com os serviços prestados, porém alguns aspectos podem ser ainda aprimorados para atender melhor às expectativas dos pacientes. Trata-se de população economicamente carente, com baixa acuidade visual, dependente do serviço público de saúde e apresentando baixo nível de instrução; além disso, a maioria tem necessidade de acompanhante durante as visitas.

Descritores: Satisfação do paciente; Catarata; Promoção da Saúde; Saúde Pública; Questionários

Aluna do curso MBA - Administração em Saúde da Universidade Federal de São Paulo - UNIFESP.

${ }^{2}$ Professora afiliada e Livre Docente do Departamento de Oftalmologia da Universidade Federal de São Paulo - UNIFESP.

${ }^{3}$ Professor Titular do Departamento de Oftalmologia da Universidade Federal de São Paulo - UNIFESP.

Endereço para correspondência: Luci Meire Pereira da Silva, Rua Botucatu 822 - São Paulo (SP)

CEP 04023-062 - E-mail: luci@ oftalmo.epm.br

Recebido para publicação em 07.05.2003

Versão revisada recebida em 04.03.2004

Aprovação em 18.05.2004

\section{INTRODUCÃO}

A perda da visão é um dos mais sérios infortúnios que podem suceder a uma pessoa. De acordo com a OMS/WHO (Organização Mundial da Saúde/ World Health Organization), existem atualmente, aproximadamente, $180 \mathrm{mi}-$ lhões de deficientes visuais, sendo que 50 milhões de pessoas são classificadas como cegas. Estimou-se, no ano de 2000, que 8 milhões de casos novos de cegueira estariam ocorrendo anualmente, no mundo, deixando claro que este número poderia ser duplicado nos anos subseqüentes, se programas de saúde pública não fossem intensificados, desenvolvidos e implantados ${ }^{(1-2)}$.
\end{abstract}


Dependendo da causa, até $80 \%$ dos casos de cegueira ou deficiência visual podem ser evitados, seja por mecanismos de prevenção, seja por realização de tratamento específico ${ }^{(1,3)}$.

Cegueira é definida, pela WHO/OMS - Organização Mundial de Saúde, conforme tabela CID-10 - Classificação Internacional de Doenças - Décima Revisão, como acuidade visual (AV) menor do que 20/400 (3/60; 0,05 [Tabelas Snellen e ETDRS]), no melhor olho, com a melhor correção possível, ou como uma perda de campo visual, em cada olho, para menos de $10^{\circ}$, a partir da fixação ${ }^{(4)}$.

Baixa acuidade é definida como acuidade visual (AV) menor do que 20/70 (6/18; 0,3 [Tabelas Snellen e ETDRS]), mas igual, ou melhor, do que 20/400, com a melhor correção possível ${ }^{(4)}$.

A catarata é a principal causa mundial de cegueira, sendo responsável por $50 \%$ dos casos de incapacidade visual ${ }^{(1)}$. Há um número estimado de 17 milhões de cegos por catarata no mundo, dos quais 10 milhões estão somente na Índia ${ }^{(3,5-6)}$, onde a incidência anual é de aproximadamente 3,8 milhões ${ }^{(7-8)}$.

Estima-se que a prevalência de cegueira por catarata deva ser duplicada até o ano de 2010, em razão da crescente expectativa de vida resultando no aumento da população idosa no mundo ${ }^{(5-6,9)}$.

Pessoas idosas têm maior chance de desenvolver catarata e a maioria das pessoas com catarata estão acima dos 50 anos de idade. Isto significa que com o aumento da expectativa de vida, mais pessoas irão desenvolver catarata e o número de casos de cegueira irá aumentar. Segundo dados da OMS, em 2020 haverá 1,2 bilhões de pessoas com 60 anos ou mais no mundo ${ }^{(1,9)}$.

Em 1998, no Brasil, foi estimada a existência de 600 mil pessoas cegas por catarata, que se encontravam em duas situações básicas: sem diagnóstico estabelecido; ou com diagnóstico, à espera da realização do procedimento cirúrgico. Além disso, estimava-se que, a cada ano, haveria cerca de $120 \mathrm{mil}$ novos casos de catarata no país, constituindo-se assim, a catarata como a maior causa de cegueira reversível no Brasil( ${ }^{(10)}$.

De acordo com o Conselho Brasileiro de Oftalmologia (CBO), estima-se que aproximadamente $1 \%$ da população brasileira seja deficiente visual e que as principais causas de cegueira sejam vícios de refração não corrigidos e catarata. De acordo com dados estatísticos do CBO, existem, no nosso país, cerca de 350 mil pacientes acima de 50 anos, que estão cegos devido à catarata, sendo que, em 95\% destes casos, a cegueira pode ser eliminada ${ }^{(11)}$.

Até o início do mutirão de cirurgias eletivas, campanha lançada pelo Ministério da Saúde em 1999, o número médio de cirurgias de catarata realizadas por ano, através do SUS, era de aproximadamente 100 mil. Neste ritmo de atendimento de rotina, se considerada a lista de espera então existente, a demora para realização do tratamento cirúrgico poderia se estender por cerca de sete anos e se agregarmos a essa lista os casos novos surgidos a cada ano, seria fácil prever uma fila interminável. Como resultado destes mutirões, já foram realizadas cerca de 753.078 cirurgias de catarata, no período de maio de 1999 até fevereiro de $2002^{(10,12)}$.
Ainda, segundo o Conselho Brasileiro de Oftalmologia (CBO), o caminho para a resolução de tal problema exige um planejamento em 4 setores: educação da população em saúde ocular; priorização do atendimento e uso de tecnologia apropriada; aumento de verbas para a saúde de modo geral; trabalho conjunto entre especialistas, autoridades e usuários ${ }^{(11)}$.

Desde 1992, O Instituto da Visão/Departamento de Oftalmologia da UNIFESP vem desempenhando importante papel na melhora do atendimento oftalmológico para uma população numerosa de idosos cegos, sem condições financeiras e sem acesso a tratamento adequado ${ }^{(11)}$.

Em 1994, aconteceu a $1^{\underline{a}}$ Campanha Nacional de Reabilitação Visual do Idoso do Departamento de Oftalmologia da UNIFESP. As campanhas de mutirão tornaram possível ampliar a oferta de serviços e acesso a procedimentos cirúrgicos eletivos e, conseqüentemente, reduzir filas, tempo de espera, melhorando significativamente a qualidade de vida daqueles que eram precocemente aposentados devido à incapacidade visual e, assim, excluídos da sociedade ${ }^{(3,11)}$.

Graças a um esforço conjunto do Instituto da Visão/ UNIFESP, Ministério da Saúde em parceria com o CBO e entidades que prestam serviços voluntários, o problema de catarata, que é a grande causa de cegueira evitável e reversível no nosso país, está sendo rapidamente equacionado e já é possível acreditar que, em breve, o Brasil será o primeiro país da América Latina a deixar de ter filas de pacientes cegos aguardando cirurgia de catarata ${ }^{(10,12)}$.

Desde o início dos Mutirões do Departamento de Oftalmologia da UNIFESP, já foram atendidas mais de 80 mil pessoas, não só da cidade de São Paulo, mas também de municípios próximos a grande São Paulo, do interior de São Paulo e até mesmo de estados distantes da região norte e nordeste do país. Estas campanhas já promoveram cirurgias de catarata em aproximadamente 16.000 pacientes, que foram atendidos e tratados gratuitamente ${ }^{(13)}$.

Acrescenta-se aos saldos positivos dos Mutirões, relacionados à solução da demanda reprimida, o fato de que estas campanhas têm desempenhado um importante papel na organização da assistência de rotina, além de educar, propagando informações sobre a doença e suas formas de tratamento, e de estimular a procura por serviços de saúde, mesmo nos períodos não contemplados por campanhas ${ }^{(10)}$.

O Departamento de Oftalmologia da UNIFESP vem se esforçando para minimizar o problema de cegueira provocado pela catarata, investindo na formação de profissionais e na ampliação das áreas de atendimento oftalmológico gratuito, além de participar ativamente do planejamento e execução de Campanhas de prevenção e reabilitação visual junto ao $\mathrm{CBO}^{(11,14)}$.

O objetivo deste estudo foi identificar o perfil socioeconômico e epidemiológico, bem como avaliar a satisfação dos pacientes atendidos durante os Mutirões de Catarata do Instituto da Visão - Departamento de Oftalmologia - UNIFESP. 


\section{MÉTODO}

O método deste estudo foi observacional.

A amostra foi formada por 133 sujeitos atendidos no Mutirão de Catarata realizado em 5 de outubro de 2002, no Departamento de Oftalmologia da Universidade Federal de São Paulo UNIFESP, representando 50\% do atendimento, recrutados alternadamente e aleatoriamente, na fila de espera.

Para o propósito deste estudo utilizou-se questionário, com a finalidade de coletar dados epidemiológicos (Anexo 1) e avaliar a satisfação dos pacientes com relação ao atendimento (Anexo 2). Os questionários foram desenvolvidos pelos próprios investigadores, especificamente para este estudo, baseados em modelos padronizados já utilizados em estudos similares realizados no Brasil e no exterior ${ }^{(15-20)}$.

Para assegurar a confiança, credibilidade e compreensão, os questionários foram testados previamente em população com características semelhantes às da população-alvo do estudo. Também a equipe que realizou as entrevistas foi treinada e o método de abordagem aos pacientes, da formulação das questões e dos registros das respostas foi padronizado ${ }^{(21)}$.

Para caracterizar a amostra foram selecionadas as seguintes variáveis: sexo, idade, raça/etnia, nível de instrução, estado civil, tipo de atividade profissional, renda familiar mensal e região de residência. Foram registrados dados sobre o tempo decorrido desde o último exame oftalmológico e o tipo de serviço que foi utilizado.

Após a assinatura do Termo de Consentimento Livre e Esclarecido, com ajuda de voluntários que orientavam quanto ao preenchimento e esclareciam eventuais dúvidas, foi aplicado o questionário 1 para identificar o perfil socioeconômico e coletar dados epidemiológicos dos pacientes (Anexo 1). Além das respostas do questionário, foram anotadas também acuidade visual e doença ocular.

Ao final do atendimento, foi aplicado o questionário 2 para avaliar o grau de satisfação de todos os voluntários selecionados previamente. Este questionário foi composto por questões abertas e fechadas, com respostas de múltipla escolha (Anexo 2).

\section{RESULTADOS}

Com relação ao perfil socioeconômico e epidemiológico (Gráfico 1), as principais características encontradas foram:

- $102(76 \%)$ eram provenientes de SP-Capital, 23 (17\%), da grande São Paulo, 4 (4\%), de cidades do interior e litoral de São Paulo e 4 (3\%), de outros estados;

- 61 (46\%) eram homens e 72 (54\%), mulheres;

- do total dos entrevistados 117 (87\%) tinham mais de 50 anos de idade;

- 84 (62\%) eram brancos, 39 (29\%), pardos, 09 (7\%), negros e 2 (2\%), amarelos;

- 66 (49\%) eram casados, 38 (29\%), viúvos, 13 (10\%), solteiros e 16 (12\%), divorciados ou separados;
- 101 (84\%) pacientes tinham como nível de instrução apenas o primeiro grau;

- $74(55 \%)$ eram aposentados ou pensionistas, $3(2 \%)$, desempregados, 6 (5\%), autônomos, 5 (4\%), comerciários, $24(18 \%)$, do lar e 21 (16\%) tinham outras atividades.

- $99(74 \%)$ tinham renda familiar até $\mathrm{R} \$ 500,00$

Também foram identificadas outras características, que estão descritas a seguir:

- $52(39 \%)$ realizaram exame oftalmológico há menos de 1 ano, 46 (35\%), há 1-2 anos, 27 (20\%), há 3 anos ou mais e $8(6 \%)$ nunca haviam realizado exame oftalmológico;

- $77(58 \%)$, realizaram o último exame oftalmológico através de serviços do SUS, 17 (13\%), de convênios, 31 (23\%), de médicos particulares e 8 (6\%) nunca haviam recebido atendimento oftalmológico;

- $36(27 \%)$ tomaram conhecimento do mutirão através de TV ou rádio, 18 (14\%), no posto de saúde, 49 (36\%), por meio de parentes ou amigos, 29 (22\%), no Departamento de Oftalmologia - HSP e 1 (1\%), faixa informativa que viu na rua;

- 34 (26\%), utilizaram apenas ônibus para acesso ao local do mutirão, 44 (33\%) utilizaram metrô-ônibus, 7 (5\%), apenas Metrô; 4 (3\%), táxi, 35 (26\%), veículo próprio e 9 (7\%), outros meios de condução, que incluíam transporte do próprio posto ou prefeitura da cidade;

- 91 (68\%) tiveram necessidade de vir com acompanhante;

- $95(71 \%)$ pacientes apresentavam catarata com indicação cirúrgica, 11 (8\%) eram portadores dessa doença ocular, porém sem indicação cirúrgica no momento, 13 (10\%) não apresentavam catarata, mas sim, outras patologias que incluíam glaucoma e doenças de retina;

- $92(97 \%)$ dos que necessitam de cirurgia concordaram em se submeter a essa intervenção, 2 (2\%) não concordaram porque estavam conformados com a sua visão e $1(1 \%)$ não concordou, pois tinha medo da cirurgia.

Com relação à satisfação com o atendimento recebido durante o Mutirão (Gráficos 2 e 3), foram obtidos os seguintes resultados:

- $83(63 \%)$ consideraram bom o tempo de espera para atendimento na fila externa do Instituto da Catarata, 39 (29\%), regular e $11(8 \%)$, ruim;

- $123(92 \%)$ qualificaram como bom o pré-atendimento realizado pelo pessoal de enfermagem e atendentes e 10 (8\%) classificaram o atendimento como regular;

- $128(96 \%)$ consideraram bom o atendimento médico recebido, $4(3 \%)$, regular e $1(1 \%)$, ruim;

- $107(80 \%)$ pacientes tiveram suas dúvidas totalmente esclarecidas, $21(16 \%)$ foram parcialmente esclarecidos e 5 (4\%) consideraram que suas dúvidas não foram esclarecidas pelo médico;

- $130(97 \%)$ pacientes consideraram que receberam atenção e respeito do médico, 2 (2\%), tiveram atenção e respeito em parte e $1(1 \%)$ relatou não ter tido atenção nem respeito por parte do médico; 


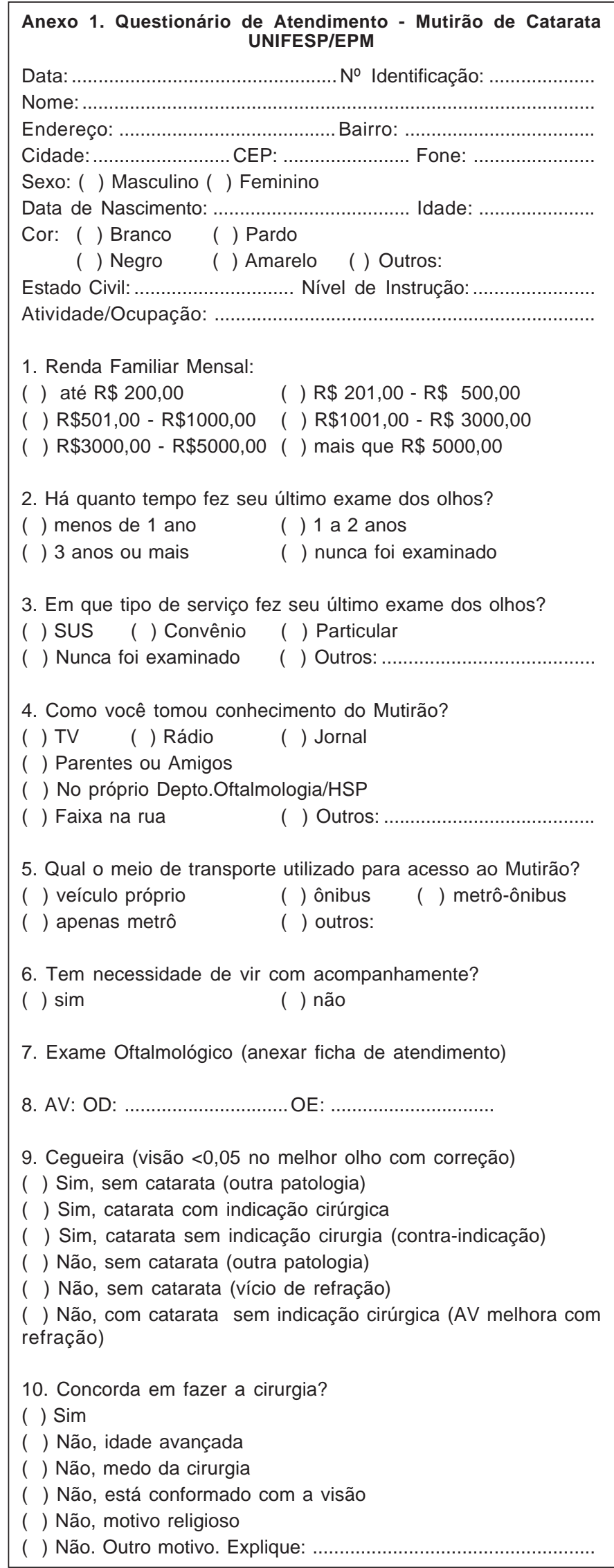

\section{Anexo 2. Questionário de Satisfação - Mutirão de Catarata} UNIFESP/EPM

Identificação (iniciais):

Idade: Sexo: ( )F ( )M

Data: .

1. O que você achou do tempo de espera na fila do lado de fora do Hospital?

( ) Bom ( ) Regular ( ) Ruim. Por quê?:

2. O que você achou do pré-atendimento feito pelo pessoal da enfermagem e atendentes?

( ) Bom ( ) Regular ( ) Ruim. Por quê?:

3. O que você achou do tratamento médico recebido?

( ) Bom ( ) Regular ( ) Ruim. Por quê?:

4. Todas as suas dúvidas quanto ao tratamento foram esclarecidas pelo médico?

( ) Sim ( ) Em parte ( ) Não. Por quê?:

5. Você recebeu atenção e respeito do médico que o atendeu?

( ) $\operatorname{Sim}($ ) Em parte ( ) Não. Por quê?:

6. A linguagem utilizada pelo médico foi clara?

( ) $\operatorname{Sim}($ ) Em parte ( ) Não. Por quê?:

7. O que você achou da qualidade geral do atendimento?

( ) Boa ( ) Regular ( ) Ruim. Por quê?:

8. As suas expectativas foram atendidas, você ficou satisfeito? ( ) Sim ( ) Em parte ( ) Não. Por quê?:

9. O que você achou da organização do mutirão?

( ) Boa ( ) Regular ( ) Ruim. Por quê?:

10. O que você achou do ambiente físico do Hospital (salas, móveis e equipamentos)

( ) Bom ( ) Regular ( ) Ruim. Por quê?:

11. Qual o tempo total que você esperou para concluir o atendimento?

( ) 1 a 2 horas

( ) 2 a 3 horas

( ) 3 a 4 horas

( ) mais do que 4 horas

12. Você já havia tentado alternativas para atendimento nesta especialidade?

( ) Sim. Quais:
( ) Posto de Saúde
( ) Consultórios particulares
( ) Hospitais Públicos
( ) Rede de Convênios
( ) Outros. Especifique:

\section{( ) Não}

13. Você tem algum tipo de convênio médico:

( ) sim. Por que não utilizou:

( ) não 

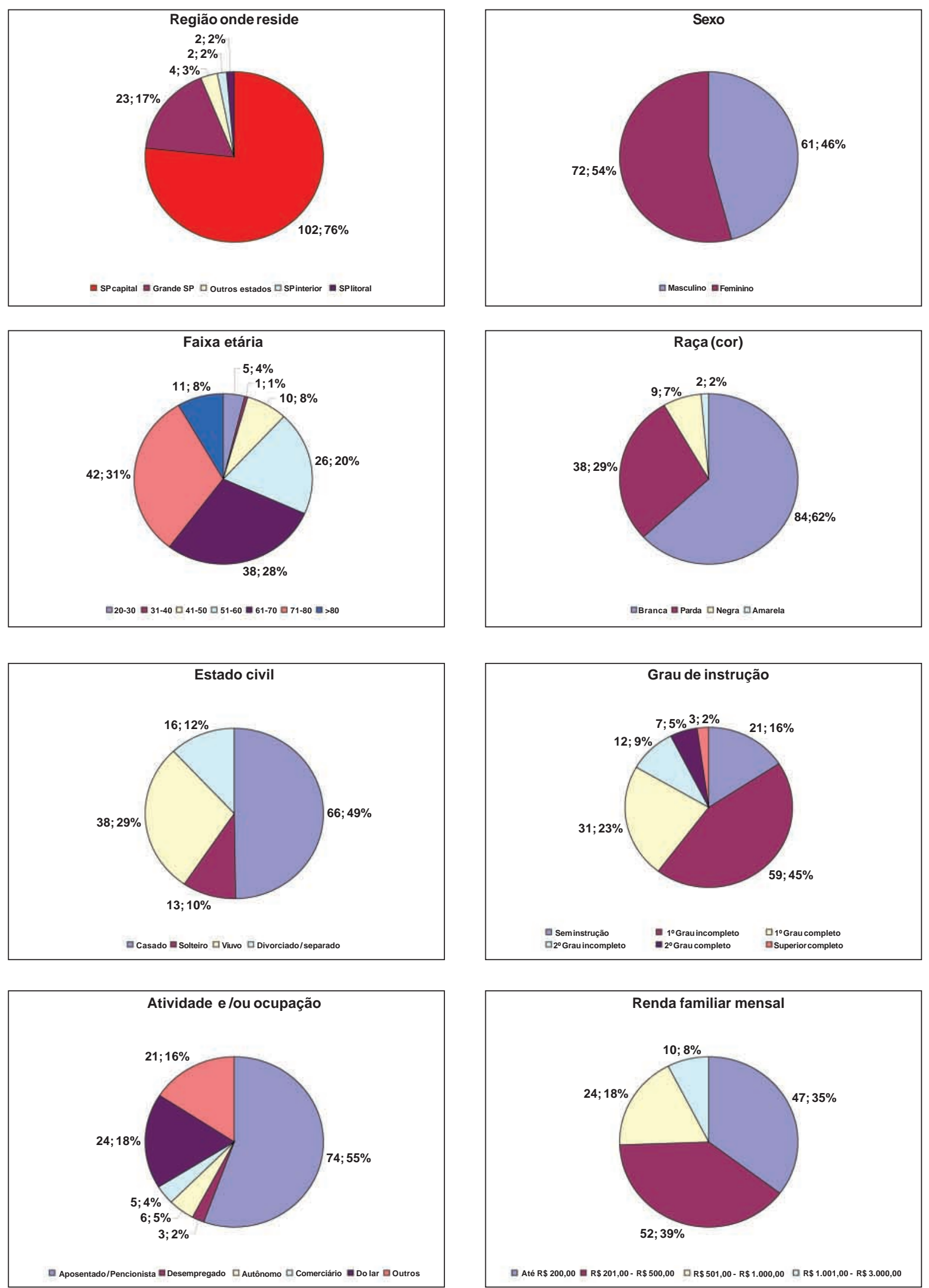

Gráfico 1 - Perfil socioeconômico - Principais características $(n=133)$ 
- $124(93 \%)$ concordam que a linguagem utilizada pelo médico foi totalmente clara e $9(7 \%)$ a consideraram parcialmente clara;

- $122(91 \%)$ classificaram como boa a qualidade geral do atendimento, $10(8 \%)$, regular e $1(1 \%)$, ruim;

- $109(81 \%)$ tiveram suas expectativas totalmente atendidas e ficaram satisfeitos, 18 (14\%) tiveram suas expectativas parcialmente atendidas e $6(5 \%)$ não tiveram suas expectativas atendidas e não ficaram satisfeitos;

- $108(81 \%)$ consideraram boa a organização do mutirão, $23(17 \%)$, regular e $2(2 \%)$, ruim;

- $123(92 \%)$ acharam bom o ambiente físico do INCAT (sala de espera, móveis, equipamentos, etc), 10 (8\%), regular e nenhum paciente o considerou ruim;

- $40(30 \%)$ aguardaram entre 1-2 horas para concluir atendimento, 53 (40\%), entre 2-3 horas, 27 (20\%), entre 3-4 horas e 13 (10\%) aguardaram mais do que 4 horas;

- $45(34 \%)$ pacientes procuraram alternativas antes de optarem pelo mutirão e 88 (66\%) optaram diretamente pelo atendimento no mutirão;

- Dos pacientes que tentaram alternativas antes de optarem pelo mutirão, 42 (47\%) procuraram hospitais públicos ou universitários, 29 (33\%), postos de saúde, 11 (13\%), rede credenciada de convênios e 6 (7\%), consultórios particulares.

- $122(92 \%)$ pacientes não possuíam convênio médico e $11(8 \%)$ possuíam convênio médico. Dentre estes, 10 (91\%) não utilizaram o convênio porque não havia cobertura da cirurgia ou da lente intra-ocular e 1 (9\%), devido à demora e burocracia para agendar cirurgia.

Foram registradas, esporadicamente, como principais causas de insatisfação manifestada por aproximadamente $10 \%$ dos pacientes atendidos, as seguintes queixas:

- Falta de cadeiras para idosos que têm dificuldade de esperar em pé;

- Incômodo para pingar colírio no sol;

- Falta de lugar coberto para abrigar do sol e da chuva, durante espera na fila;

- Demora no atendimento;

- Demora no agendamento dos exames pré-operatórios;

- Falta de triagem na fila, priorizando aqueles que apresentam estado de saúde mais debilitado ou idade muito avançada;

- Pouco espaço físico no INCAT;

- Falta de fiscalização para evitar pessoas "fura-fila";

- Insuficiente o suprimento de papel higiênico nos banheiros;

- Atendimento muito impessoal;

- Falta de orientação clara para encaminhamento aos outros setores e para retornos.

\section{DISCUSSÃO}

Os atendimentos de mutirão têm se mostrado como impor-

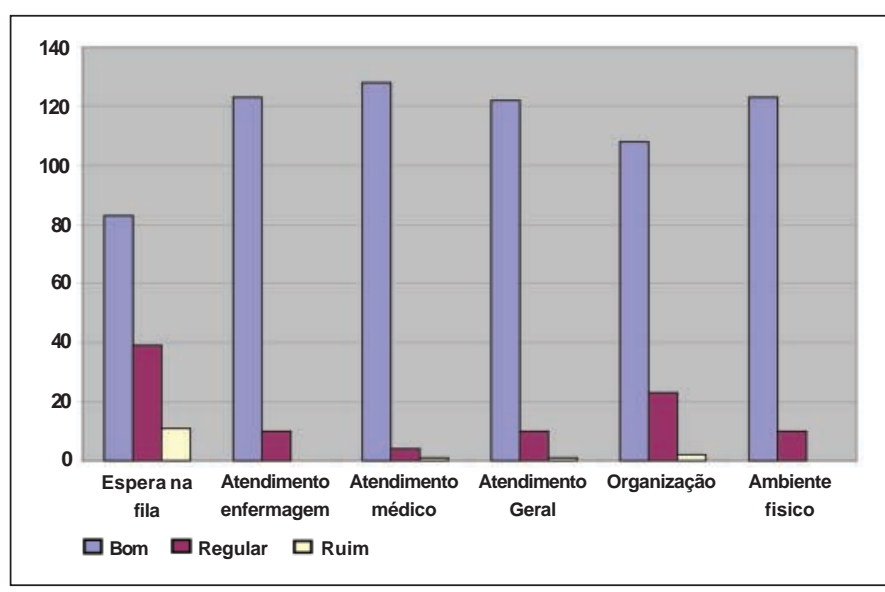

Gráfico 2 - Opinião dos pacientes quanto ao atendimento e organização

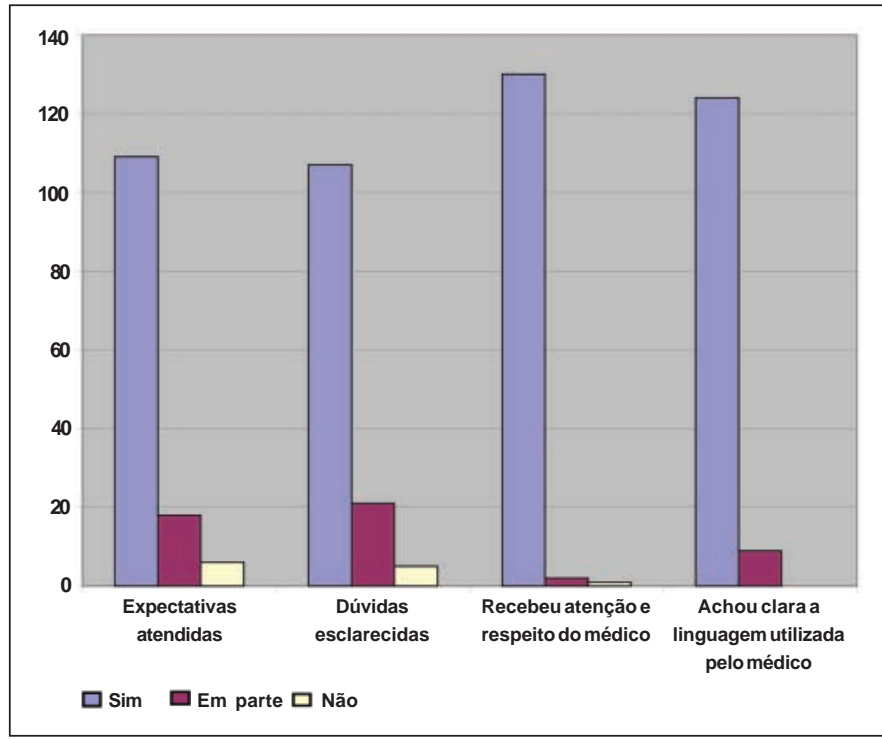

Gráfico 3 - Opinião dos pacientes quanto ao atendimento das expectativas, esclarecimentos de dúvidas, atenção, respeito e linguagem utilizada pelo médico

tante recurso para aumentar significativamente o número de cirurgias de catarata realizadas em instituições públicas, reduzindo a demanda reprimida dos casos tratáveis de cegueira no país, através de prescrição de óculos para correção de erros refracionais, realização de cirurgias de catarata, educação e conscientização da população( ${ }^{(5)}$.

Embora os mutirões não apresentem condições ideais de atendimento, devido ao volume de pessoas e ao tempo de espera, sem o conforto encontrado nos serviços privados, 122 (91\%) pacientes classificaram como bom o atendimento geral recebido durante o Mutirão de Catarata do Instituto da VisãoDepartamento de Oftalmologia da UNIFESP, mesmo considerando que $70 \%$ tiveram de esperar de 1 a 3 horas para receber $\mathrm{o}$ atendimento.

A partir dos 50 anos de idade, aumenta o risco de desenvolvimento de doenças oculares e sistêmicas, tornando mais 
caro os cuidados com a saúde para essa faixa etária. Estima-se que $80 \%$ da população dependem de assistência médica governamental $^{(5)}$. Foi constatado, neste estudo, que $36 \%$ dos sujeitos já tiveram condições de atendimento pelo setor privado e agora dependem do atendimento na rede pública de saúde, evidenciando, assim, a piora da situação socioeconômica da população e o conseqüente aumento da procura pelo serviço público de saúde.

Conforme dados encontrados neste e em outros estudos similares $^{(16,18-19)}$, foi observado que a população atendida nos projetos de Mutirões é economicamente carente e possui baixa acuidade visual; são pacientes que dependem, em sua maioria, do serviço público de saúde e que apresentam baixo nível de instrução; a maior parte deles requer acompanhante e necessita se deslocar grandes distâncias para ter acesso ao atendimento. Assim sendo, constata-se que os Mutirões vão de encontro ao objetivo proposto, que é justamente atender e assistir a população carente e sem outro recurso disponível.

Foi possível ainda detectar que $6 \%$ dos participantes, apesar de idosos, nunca haviam recebido tratamento oftalmológico. Este fato foi considerado relevante, uma vez que a cidade de São Paulo tem ampla rede de atendimento e grande quantidade de recursos. Foi identificado também que $58 \%$ da população deste estudo, embora tivessem tido acesso à consulta através de outros serviços de saúde, não conseguiram submeter-se à cirurgia e recorreram ao atendimento do Mutirão.

Portanto, vale a pena ressaltar que, apesar do importante papel dos Mutirões na melhoria do acesso a tratamento oftalmológico, é necessário também garantir o atendimento continuado, através da rede pública, a toda a população.

Levando em consideração o perfil socioeconômico dos pacientes, é importante evitar que estes tenham que se deslocar, inúmeras vezes, para os locais de consulta, pois essa dificuldade pode se tornar um obstáculo para idosos, deficientes visuais de baixa renda e com saúde debilitada. Para maior eficiência, o paciente deveria fazer todos os procedimentos no mesmo dia e no local das campanhas, inclusive os exames préoperatórios (coleta de sangue, eletrocardiograma, radiografia de tórax, avaliação pré-anestésica-APA) e o agendamento da cirurgia, evitando, assim, deslocamentos desnecessários, o que resultaria em economia para os pacientes e para o sistema de saúde.

Estudo já desenvolvido ${ }^{(22)}$ sugere que a realização de exames médicos de rotina, antes da cirurgia de catarata, não reduz a taxa de complicações durante o pós-operatório. Assim sendo, talvez fosse mais eficiente não solicitar estes exames préoperatórios de rotina, a não ser que indicado pela história do paciente ou pelo exame físico.

Devido à escassez de dados epidemiológicos sobre a catarata no Brasil, o desenvolvimento e utilização de fichas e questionários específicos para os mutirões devem possibilitar coleta de dados e conhecimento das condições de cegueira, bem como apurar os resultados das cirurgias e o impacto na qualidade de vida destes pacientes.
Para que a população possa ter amplo acesso às informações sobre tratamentos e educação ocular, seria importante intensificar a divulgação do calendário das campanhas através de meios de comunicação de massa (Rádio, TV, jornais, folhetos distribuídos em locais de aglomeração, etc), juntamente com a distribuição de material educativo sobre saúde ocular, catarata e formas de tratamento e prevenção.

Também vale ressaltar a importância de se realizar uma avaliação ao final de cada projeto, divulgando os resultados obtidos e relacionando sugestões para melhorias futuras.

\section{CONCLUSÃO}

Diante dos resultados apurados, concluiu-se que a população atendida no Mutirão de Catarata do Instituto da Visão Departamento de Oftalmologia - UNIFESP ficou satisfeita com os serviços prestados, porém alguns aspectos podem ser aprimorados para atender melhor às expectativas dos pacientes. Trata-se de uma população economicamente carente, com baixa acuidade visual que depende principalmente do serviço público de saúde e apresenta baixo nível de instrução, além de, em sua maioria, ter necessidade de acompanhante.

\section{ABSTRACT}

Objective: To identify the socioeconomic profile as well as epidemiological data and evaluate the satisfaction of patients examined during a community project for the treatment of senile cataracts conducted by the Instituto da Visão/Ophthalmology Department - UNIFESP-EPM. Method: During the Cataract Project which occurred on $5^{\text {th }}$ Sept, 2002 at the Ophthalmology Department - UNIFESP/EPM, subjects were surveyed by questionnaires developed to identify their socioeconomic characteristics and evaluate their satisfaction regarding attendance. They were randomly selected from the waiting line. Results: The sample was composed of 133 subjects, which represents $50 \%$ of patients examined during this Cataract Project. Sixty-one (46\%) were male and 72 (54\%) female; $117(87 \%)$ were older than 50 years; 111 (84\%) finished high school; 74 (55\%) were retired and/or pensioners; 99 $(74 \%)$ had a family income of up to $\mathrm{R} \$ 500,00 ; 91(68 \%)$ needed someone to assist or accompany them during the visit; 95 (71\%) presented cataract with surgical indication. Regarding their satisfaction with the service, $122(91 \%)$ considered the general quality of attendance good; $10(8 \%)$ considered it regular and 1 (1\%) bad. Conclusion: As a whole the population examined during this Cataract Project was satisfied with the services, but, some aspects have to be improved to better meet their expectations. It is a needy population, with low visual acuity, depends on public health services, has low level education and, most, need someone to accompany them during the visit to the hospital.

Keywords: Patient satisfaction; Cataract; Health Promotion; Public Health; Questionnaires 


\section{REFERÊNCIAS}

1. World Health Organization [homepage na Internet]. Blindness: Vision 2020 Global Initiative for the Elimination of Avoidable Blindness [updated 2000 Fev; cited 2003 Fev 13]. WHO, Department of Chronic Diseases and Health Promotion (CHP); [about 8 screens]. Available from: http://www.who.int/ mediacentre/factsheets/fs213/em/

2. World Health Organization [homepage na Internet]. Prevention of blindness and deafness. [cited 2003 Fev 13]. WHO, Department of Chronic Diseases and Health Promotion (CHP); [about 2 screens]. Available from: http://www.who. int/pdb/pdl/pdl_home.html

3. Kara-José (organizador). Prevenção da cegueira por catarata. Campinas, UNICAMP, 1996.

4. Classificação Internacional de Doenças [monograph on the Internet]. Brasília: Ministério da Saúde; c1993. [10ª revisão; citado em 2003 Fev 13]. Disponível em http://www.cid10.hpg.ig.com.br/index.htm

5. Kara-José N, Temporini ER. Catarata e Cegueira - Epidemiologia e Prevenção. In: Arieta CEL. Cristalino e catarata. Rio de Janeiro: Cultura Médica, 2002. p.50-6

6. Sommer A. Public health aspects of ophthalmic disease: introduction.. Annu Rev Public Health. 1996;17:115-9. Review.

7. Kupfer C. A decade of progress in the prevention of blindness. In: Kupfer C, editor. World Blindness and Its Prevention. London: Oxford University Press; 1988. v.3, p.58-69.

8. Minassian DC, Mehra V. 3.8 million blinded by cataract each year: projections from the first epidemiological study of incidence of cataract blindness in India. Br J Ophthalmol. 1990;74(6):341-3.

9. Javitt JC, Wang F, West SK. Blindness due to cataract: epidemiology and prevention. Annu Rev Public Health. 1996:17:159-77. Review.

10. Ministério da Saúde [página na Internet]. Redução de demandas reprimidas: mutirões. [citado em 2002 Set 4]. Ministério da Saúde (SAS); [cerca de 3 telas]. [Disponível em: http://saude.gov.br/sas/relatorio/6.4\%20demandas.htm,
11. Catarata: parceria e soluções. Instituto da Catarata. Universidade Federal de São Paulo - Escola Paulista de Medicina e Fundação Lions do Distrito L4, Lions International.

12. Mutirão de catarata realiza 753 mil cirurgias. Informe Saúde 2002;6(162).[não paginado]. [Publicação da Divisão de Jornalismo da Assessoria de Comunicação Social do Ministério da Saúde].

13. Belfort Jr R. Nota divulgada para imprensa sobre a realização do $40^{\circ}$ Mutirão do Instituto da Visão - UNIFESP/EPM, realizado em 3/2/02. [citado em 2003 Fev 13]. Disponível em: www.portalweb01.saude.gov.br/saude/aplicacoes/informe/arquivos/informe162.pdf

14. Atividades do Instituto da Catarata. Boletim Informativo da Fundação Lions do Distrito LC-2 2001;(2):17-9.

15. Margolis SA, Al-Marzouq S, Revel T, Reed RL. Patient satisfaction with primary health care services in the United Arab Emirates. Int J Qual Health Care. 2003;15(3):241-9.

16. Kara-Junior N, Temporini ER, Kara-José N. Cataract surgery: expectations of patients assisted during a community project in Sao Paulo, state of São Paulo, Brazil. Rev Hosp Clin. 2001;56(6):163-8.

17. Kotaka F, Pacheco MLR, Higaki Y. Avaliação pelos usuários dos hospitais participantes do programa de qualidade hospitalar no Estado de São Paulo, Brasil. Rev Saúde Pública. 1997;31(2):171-7.

18. Kara-José N, Temporini ER. Cataract surgery: or why are there some patients excluded. Rev Panam Salud Publica. 1999;6(4):242-8. id:pt.

19. Temporini ER, José NK, José Júnior NK. Catarata senil: características e percepções de pacientes atendidos em projeto comunitário de reabilitação visual. Arq Bras Oftalmol. 1997;60(1):79-83.

20. Hendriks AA, Oort FJ, Vrielink MR, Smets EM. Reliability and validity of the Satisfaction with Hospital Care Questionnaire. Int J Qual Health Care. 2002;14(6):471-82

21. Piovesan A, Temporini ER. Pesquisa exploratória: procedimento metodológico para o estudo de fatores humanos no campo da saúde pública. Rev Saúde Pública.1995;29(4):318-25.

22. Lira RP, Nascimento MA, Moreira-Filho DC, Kara-José N, Arieta CE. Are routine preoperative medical tests needed with cataract surgery? Rev Panam Salud Publica. 2001;10(1):13-7.

\title{
IV SIMPÓSIO DE OFTALMOLOGIA IVO CORRÉA MEYER
}

\section{2 e 13 de novembro de 2004 Porto Alegre - RS}

\section{PROMOC̣ÃO}

\section{Associação dos Ex-alunos do Curso Ivo Corrêa Meyer}

\section{INFORMAÇÕES:}

\author{
Fone/Fax: (51) 3346-3423 e 3395-4868 \\ E-mail:iicm@terra.com.br
}

